\title{
High-Harmonic Spectroscopy: From Femtosecond to Attosecond Molecular Dynamics
}

\author{
Hans Jakob Wörner*
}

\begin{abstract}
The principles of high-harmonic spectroscopy (HHS) are illustrated using two recent examples from the literature. The method can be applied as a probe to measure the evolution of the electronic structure of a molecule during a chemical reaction on the femtosecond time scale. Alternatively, it can be used as a comprehensive method unifying pump and probe steps into a single laser cycle to measure electronic dynamics on the attosecond time scale.
\end{abstract}

Keywords: High-harmonic spectroscopy

\section{Introduction}

Time-resolved measurements of molecular and chemical dynamics have long been limited to several femtoseconds ( $\left.1 \mathrm{fs}=10^{-15} \mathrm{~s}\right)$. This fundamental limit arose from the optical period of the carrier wave. In the past decade, new techniques have emerged that offer attosecond temporal resolution (1 as $\left.=10^{-18} \mathrm{~s}\right) .^{[1,2]}$ They extend time-resolved chemistry from the atomic to the electronic time scale and provide the opportunity to study unexplored phenomena in the time domain.

The valence shell of molecules is characterized by typical energy intervals of a few electron-volts $(\mathrm{eV})$, implying that valence-shell dynamics will be observable with light or electron pulses around and below 1 fs. The last few years have seen first successes in the observation of electronic dynamics on this time scale. ${ }^{[3-7]}$ These techniques will enable a new experimental access to electronic structure and electron correlation, which is one of the most challenging problems in chemistry and physics.

All of these new techniques with attosecond temporal resolution rely on the

\footnotetext{
${ }^{\star}$ Correspondence: Prof. Dr. H. J. Wörner ETH Zürich

Laboratory for Physical Chemistry

Wolfgang-Pauli-Strasse 10

$\mathrm{CH}-8093$ Zürich

Tel.: +41446334412

Fax: +41446321538

E-mail: woerner@phys.chem.ethz.ch
}

interaction of a molecule with an intense femtosecond laser pulse. Most of them share a common fundamental mechanism, often summarized in a three-step model. ${ }^{[8]}$ Every time that the laser electric field reaches a maximum, a small portion of the valence electron wave function is moved into the continuum. This process can be understood as tunneling of the electron wave packet through the combined molecular and laser electrostatic potential. The unbound electron wave packet is then exposed to the laser field, and can recollide with the parent molecule close to a zerocrossing of the electric field. This phenomenon, 'laser-induced recollision', enables new measurement techniques: photorecombination leads to attosecond pulse production ${ }^{[9]}$ whereas elastic and inelastic electron rescattering offer new approaches to diffraction imaging. $\left.{ }^{2}, 10,11\right]$

In typical experiments, the electron has acquired up to $100 \mathrm{eV}$ of kinetic energy during its transit in the continuum and can release it in the form of an attosecond pulse. Since the emission takes place around each zero-crossing of the field, the described mechanism generates a train of attosecond pulses separated by half the period of the generating laser field. In the frequency domain, this emission corresponds to high-order harmonics of the fundamental photon energy $\omega(\Omega=\omega, 3 \omega, \ldots, 101 \omega$ ... etc.).

In addition to being a source of ultrashort laser pulses, high-harmonic emission also encodes information about the electronic structure of the molecule. This property arises from the de-Broglie wavelength of the recolliding electron which amounts to $\sim 1-2 \AA$ under typical conditions. Since the returning electron wave diffracts in the electrostatic molecular potential before it recombines, the high-harmonic spectrum reveals structural signatures such as twocenter interference ${ }^{[12]}$ and features of electronic structure. ${ }^{[13-16]}$

In addition, a high-harmonic spectrum also encodes dynamical information. Under precisely controlled experimental conditions, each photon energy in the spectrum is emitted by a unique electron trajectory, i.e. a unique time interval between ionization and recombination $(0-1.7$ fs for an $800 \mathrm{~nm}$ driving field). This has been used to track the motion of protons on the attosecond time scale ${ }^{[17,18]}$ and to study electron rearrangement following temporally confined ionization. ${ }^{[4-6]}$

The most unusual aspect of these new methods is certainly the possibility to measure attosecond dynamics using multifemtosecond pulses. ${ }^{[2]}$ This property originates in the extreme nonlinearity of the laser-matter interaction at high intensities. The nonlinearity allows the laser period to be subdivided. Rather than following the envelope of the pulse profile, the ionized electron follows individual oscillations of the electric field. The problem remains tractable thanks to quantum mechanical diffusion: the second, third and subsequent returns of the electron wave packet have quickly decreasing amplitudes. Since the three-step model relies on simplifying assumptions, like the single-active electron and strong-field approximations, it should only be used as a guide to the intuition and improved by accurate calculations (quantum scattering etc.) when detailed information is being retrieved.

\section{Probing Chemical Reactions}

Photochemical reaction pathways are controlled by the valence electrons of the reacting molecule. An elucidation of the 
associated mechanisms ideally requires the time-resolved observation of the rearrangement of the electronic structure as the reaction proceeds. Promising methods for this purpose thus need to be sensitive to the valence electron structure. Moreover, excitation fractions must remain low in femtosecond single-photon excitation in order to avoid multiphoton processes upon excitation. Therefore, the methods should also be sensitive enough to detect the excited species. High-harmonic spectroscopy fulfills both of these requirements.

In the spirit of the three-step model, the emitted high-harmonic field can be written as a product of three terms

$$
E_{\mathrm{HHG}}(\Omega)=a_{\text {ion }}(\Omega, \theta) a_{\text {prop }}(\Omega) a_{\text {rec }}(\Omega, \theta)
$$

where $\Omega$ is the emitted photon energy and $\theta$ characterizes the orientation of the molecule relative to the polarization of the laser field. The last step, photorecombination, is now well understood in terms of quantum scattering calculations developed in the context of photoionization. ${ }^{[15,16,19]}$ Most remarkably, the strong laser field does not lead to a measurable distortion of the electronic wave functions within the accuracy of current experiments, even in polarizable systems like xenon ${ }^{[20]}$ or in molecules with appreciable extension along the direction of the laser field like $\mathrm{CO}_{2} \cdot{ }^{[4,5]}$ The second step, propagation of the electron wavepacket, is largely dominated by the laser field and is well described by classical equations as evidenced by, e.g. the chirp of attosecond pulses.[21,22] The first two steps together produce an electron wave packet that weakly depends on the molecular orientation, whereas recombination is highly sensitive to the molecular frame. Eqn. (1) describes the case where the molecule is initially in the electronic ground state and multiphoton ionization prepares the cation in a single electronic state. We now extend the formalism to describe a photochemical reaction. ${ }^{[23,24]} \mathrm{A}$ weak femtosecond pump pulse prepares the molecule in a coherent superposition of the ground state and an excited electronic state through one-photon absorption

$$
\Psi(\Delta t)=c_{1} \psi_{1}+c_{2}(\Delta t) \psi_{2}(\Delta t)
$$

with $\psi_{i}$ being the multielectron wave function of state $i$ and $\Delta t$ the time delay after excitation. The high-harmonic field emitted from this coherent superposition can be expressed as

$$
\begin{aligned}
E_{\mathrm{HHG}}(\Omega)= & \left|c_{1}\right|^{2} E_{1,1}+\left|c_{2}\right|^{2} E_{2,2} \\
& +c_{1} c_{2} E_{1,2}+c_{2} c_{1}{ }^{*} E_{2,1}
\end{aligned}
$$

The first two contributions describe high-harmonic generation in a single electronic state and are well described by Eqn. (1). $E_{1,2}$ and $E_{2,1}$ represent 'cross terms' that are sensitive to the electronic coherence between states 1 and 2 . In addition, these contributions are also proportional to the overlap of the nuclear wave functions in the two electronic states, which vanishes quickly in the case of photodissociation. [23]

The high-harmonic emission from a photoexcited molecule can thus be understood from the coherent addition of the electromagnetic fields emitted by the ground and excited electronic states. In addition to Eqn. (1), the relative phase of the high-harmonic emission from the two states contributed by the propagation of the electron in the laser field plays a role. It is well approximated by $\Delta I \tau$ where $\tau$ is the electron transit time in the continuum. ${ }^{225]}$ This finding is intuitive since the contribution to the relative phase is essentially equal to the difference in phase accumulated by the bound state.

When a photochemical reaction is probed by HHS, the ground electronic state, $\psi_{1}$ in Eqn. (2), is essentially static, whereas $\psi_{2}$ undergoes the interesting dynamics. Hence, the measured intensity is the coherent addition $\left|E_{1,1}+E_{2,2}\right|^{2}$ of the emissions from the ground and excited states. $E_{1,1}$ can be used as a static reference to measure dynamical changes in $E_{2,2}$. This detection scheme is highly sensitive to weak emission and simultaneously probes its amplitude and phase ${ }^{[23,24]}$ which are conveniently separated using the transient grating method, [26,27] illustrated in Fig. 1a. Two synchronized replica of the pump pulse are crossed under a small angle in a supersonic expansion of the investigated molecules. The periodic modulation of the excitation fraction leads to a spatial modulation of the amplitude and phase of harmonic emission in the generating medium and results in diffraction along the direction of the modulation. ${ }^{[23]}$

Writing the emission from the ground and excited states in terms of the excitation fraction $r=\left|c_{2}\right|^{2}$ and high-harmonic amplitudes and phases, we obtain

$$
\begin{aligned}
E_{\mathrm{HHG}}(\Omega)= & (1-r) d_{\mathrm{g}} \exp \left(\mathrm{i} \phi_{\mathrm{g}}\right)+r d_{\mathrm{e}} \\
& \exp \left(\mathrm{i} \phi_{\mathrm{e}}\right)
\end{aligned}
$$

where $r=r_{\max } / 2 *(\cos (k x)+1)$ is the excitation fraction modulated between 0 and $r_{\max }$ along the spatial dimension $x$. The far-field profile that is observed on the detector is the Fourier transform of this quantity. The undiffracted high-harmonic signal is similar to a measurement carried out in collinear excitation with the same spatially averaged excitation fraction (see ref. [23] and [24]). The diffracted highharmonic intensity is proportional to $r^{2} / 4 \mid d$. $\exp \left(i \phi_{\mathrm{e}}\right)-\left.d_{\mathrm{g}} \exp \left(\mathrm{i} \phi_{\mathrm{g}}\right)\right|^{2}$, and thus represents a background-free measurement of the excited state dynamics through 1:1 interference with the ground state emission. Since the excitation fraction is determined from the known absorption cross section and the measured pump laser intensity, the relative amplitudes and phases $d_{\mathrm{e}}(\Delta t) / d_{\mathrm{g}}$ and $\phi_{\mathrm{e}}(\Delta t)$ - $\phi$ can be extracted.

The method described above has been
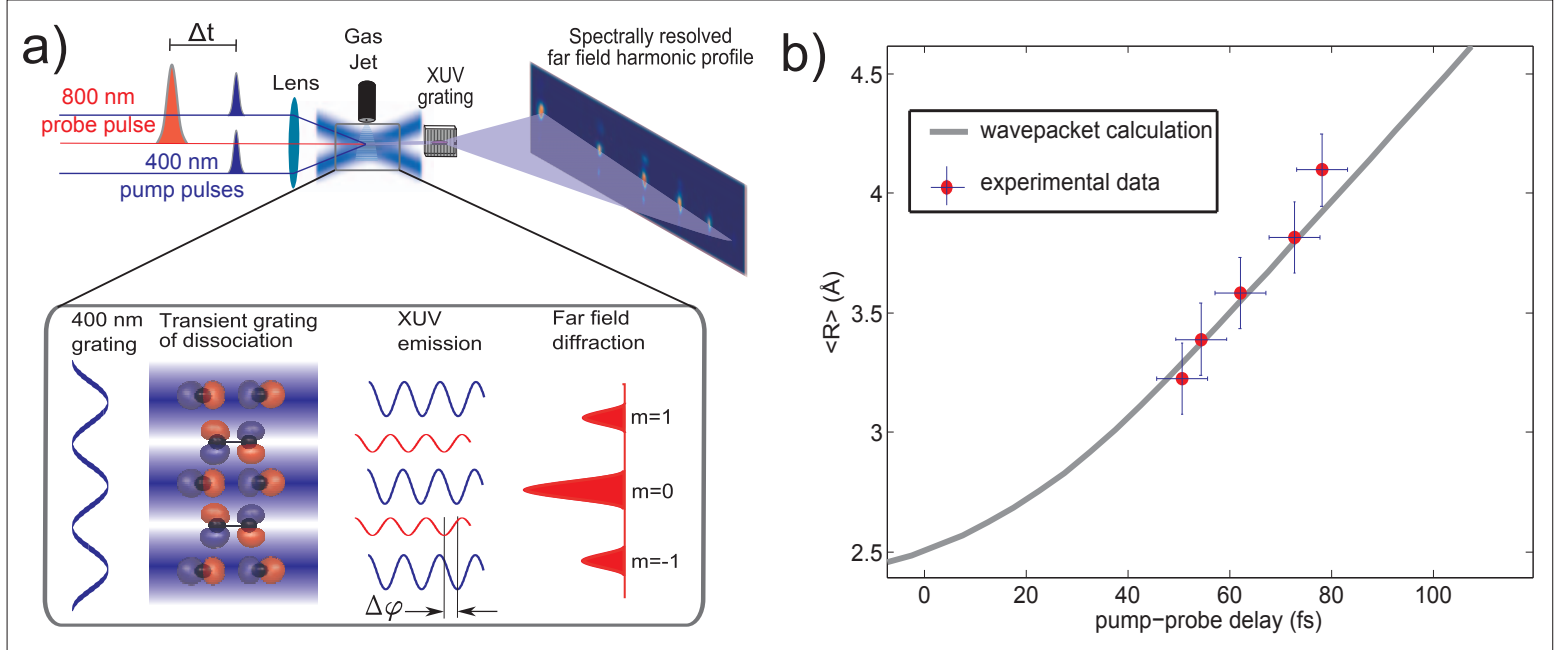

Fig. 1. High-harmonic transient grating spectroscopy. (a) Experimental setup: Two synchronized excitation pulses $(400 \mathrm{~nm})$ set up a transient grating of excitation in the molecular beam. A delayed $800 \mathrm{~nm}$ pulse generates high harmonics from the excited sample. (b) Internuclear separation of $\mathrm{Br}_{2}$ as a function of the time delay after photoexcitation to the $\mathrm{C}^{1} \Pi_{14}$ state. Data from ref. [23]. 
applied to the photodissociation of $\mathrm{Br}_{2}$ molecules.[23,24] Single-photon excitation at $400 \mathrm{~nm}$ leads to the repulsive $\mathrm{C}^{1} \Pi_{1 \mathrm{u}}$ state that dissociates into $\mathrm{Br}$ atoms in the ${ }^{2} \mathrm{P}_{3 / 2}$ ground electronic state. The relative high-harmonic amplitudes and phases for a range of harmonic orders ( $\mathrm{H} 13$ to $\mathrm{H} 21)$ have been determined from the experiment. ${ }^{[23]}$ The amplitude measurements revealed a harmonic-order dependent minimum as a function of the pump-probe time delay. These minima occur when the de-Broglie wavelength of the recombining electron wave emitting the $q^{\text {th }}$ harmonic fulfills a destructive interference condition with the internuclear separation of the molecule $R=$ $3 / 2 \lambda$. Using this relation and the measured position of the amplitude minima, one can determine the internuclear separation as a function of time as shown in Fig. 1b. In addition to the amplitudes, one also obtains the relative harmonic phases. The phases undergo a fast variation with the pumpprobe delay that reflects the variation of $I$ with the internuclear separation. The phase of the photorecombination dipole also contributes to the high-harmonic phase and characterizes the change in the electronic structure of the dissociating molecule.

\section{Probing Electronic Dynamics}

After discussing the application of HHS as a probe in femtosecond time-resolved experiments, we now turn to the attosecond time scale. High-harmonic generation can be used as a comprehensive method unifying the pump step (multiphoton ionization) and the probe step (photorecombination) into a single laser cycle. The experiment is thus repeated in each half-cycle of the laser field and the temporal information is read on the photon energy axis, exploiting the one-to-one relationship between transit time and emitted photon energy. ${ }^{[17]}$

Recent experiments show that strongfield ionization does not exclusively prepare the ground state of the cation ${ }^{[4-6,28]}$ but that excited states can also be populated. The probability of accessing a certain ionic state is exponentially sensitive to $I^{[29]}$ but also to the shape of orbitals, ${ }^{[30]}$ which can enhance the contribution of a deeply bound orbital. When ionization accesses two states of the ion and the laser-induced dynamics in the ion is neglected, highharmonic generation has two contributions

$$
E_{\mathrm{HHG}}(\Omega)=E_{1,1}+E_{2,2}
$$

which correspond to ionization from and recombination to one of the valence orbitals of the molecule. Remarkably, a cross term of the form encountered in Eqn. (3) is absent from Eqn. (4) because recombination cannot take place to an occupied orbital. The photorecombination step thus measures the phase shift accumulated by the superposition of two ionic states between ionization and recombination. However, the process is insensitive to the coherence between the two states. This differs from other recently introduced methods, like attosecond transient absorption ${ }^{[7]}$ and strong-field ionization ${ }^{[31]}$ to probe electronic dynamics.

Carbon dioxide is an instructive model system to understand multielectron effects in HHS. When $\mathrm{CO}_{2}$ is aligned parallel to the laser field generating the high harmonics, emission from HOMO of $\pi_{\mathrm{g}}$ symmetry is suppressed by the presence of a nodal plane along the direction of tunneling and recombination. HOMO-2 of $\sigma_{u}$ symmetry lies $4.3 \mathrm{eV}$ below HOMO but its emission is not suppressed by the presence of nodal planes. HOMO- 1 is of $\pi_{u}$ symmetry and is thus suppressed by both a nodal plane and a larger $I$. Thus, HHS can be described as a sum of contributions from HOMO and HOMO-2, i.e.

$$
E_{\mathrm{HHG}}(\Omega)=\sum_{i=1}^{2} \alpha_{\text {ion, } \mathrm{i}}(\Omega, \theta) \alpha_{\text {prop }, \mathrm{i}}(\Omega) \alpha_{\text {rec, } \mathrm{i}}(\Omega, \theta)
$$

Minima in $E_{\mathrm{HHG}}(\Omega)$ may thus be of structural origin and appear in $a_{\text {rec, }}(\Omega, \theta)$ or of dynamical origin and result from destructive interference in the coherent sum. These two situations can be distinguished by varying the relative phase well approximated by $\Delta I_{\mathrm{p}} \tau^{[25]}$ of the two propagation terms in Eqn. (6). For a given intensity of the laser field, the transit time $\tau$ scales approximately linearly with the wavelength $\lambda$, enabling a controlled variation of the relative phase.

Since the minimum observed in the emission from aligned $\mathrm{CO}_{2}$ molecules had been attributed to either structural[14] or dynamical origins, ${ }^{4}{ }^{4]}$ we have measured high-harmonic spectra of aligned $\mathrm{CO}_{2}$ at different wavelengths and intensities. ${ }^{[5]}$ The observed spectra are shown in Fig. 2. For a generating wavelength of $800 \mathrm{~nm}$, the minimum in aligned $\mathrm{CO}_{2}$ is observed at $42 \mathrm{eV}$, whereas at $1200 \mathrm{~nm}$, it is observed at $57 \mathrm{eV}$. This clearly shows that the minimum is not of structural origin because its position would be independent of the laser parameters, as in the case of argon. ${ }^{[6]}$ Does the observed position agree with a dynamical minimum? At $800 \mathrm{~nm}$, the relative phase accumulated by the two orbitals amounts to $\sim 3 \pi$ but at $1200 \mathrm{~nm}$, the minimum corresponds to $\sim 4.5 \pi$. Detailed calculations show that the difference arises from the photorecombination dipole moment of HOMO that undergoes a sudden phase variation at the position of the structural minimum. ${ }^{[5]}$ Thus, the minimum observed in $\mathrm{CO}_{2}$ is mostly dynamical but its position is affected by structural properties as well.

In the future, high-harmonic spectroscopy of attosecond dynamics may be operational and relevant in larger molecular systems in which the orbital relaxation upon ionization is significant. In such cases the orbitals of the neutral molecule differ from those of the ion and ionization will

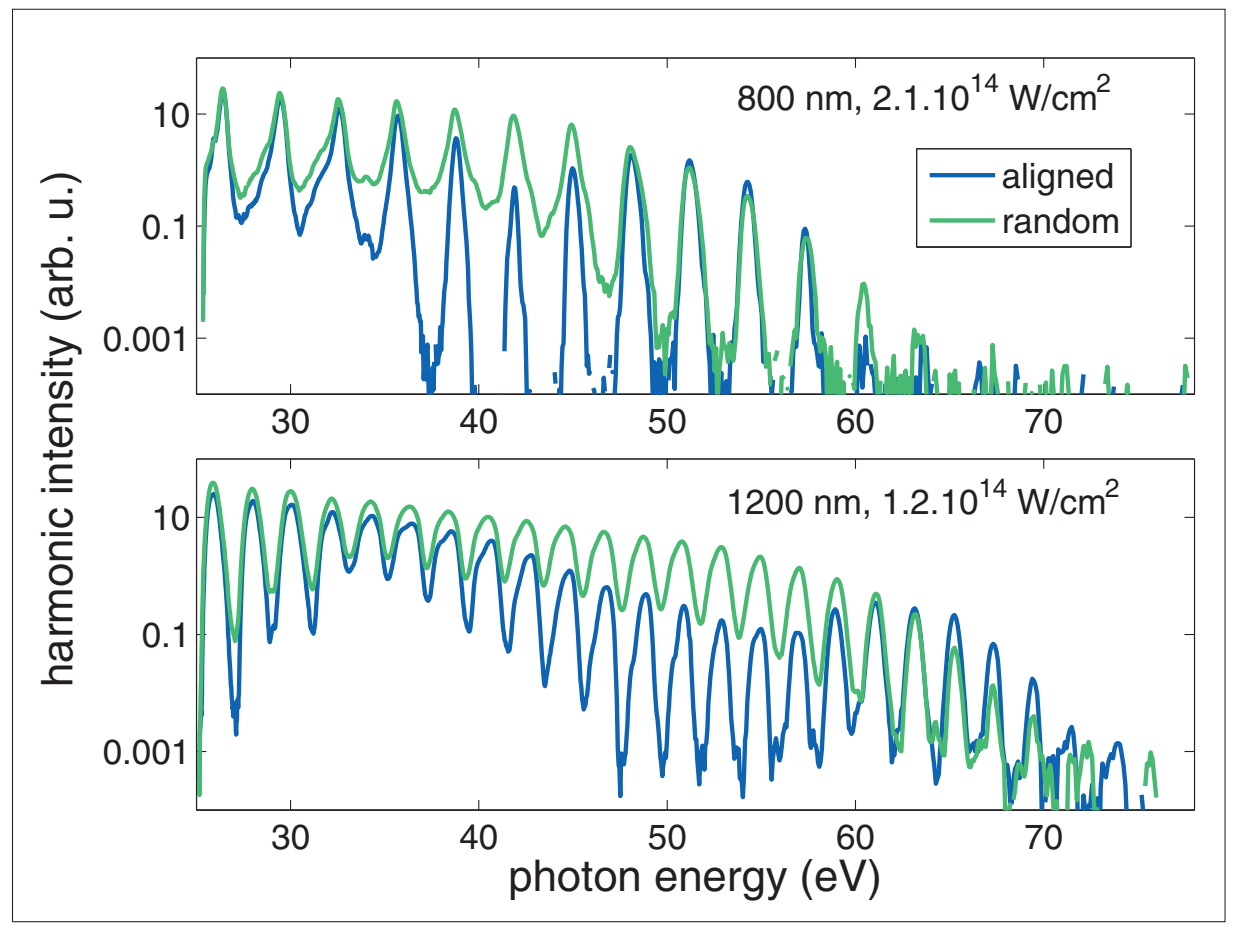

Fig. 2. High-harmonic spectroscopy of aligned $\mathrm{CO}_{2}$ molecules. The central wavelength and peak intensities of the laser pulses used to generate the high-harmonic spectra are indicated in the figures. 
trigger an electronic wave packet whose motion encodes the effects of electron correlation.

\section{Conclusions and Outlook}

The two examples discussed in this article highlight the potential of high-harmonic spectroscopy towards two different directions. The method will be applied as a probe to measure the rearrangement of the valence electronic structure of molecules undergoing chemical reactions on the femtosecond time scale. In the future, this will be particularly interesting to probe changes in electronic configurations occurring at conical intersections. High-harmonic spectroscopy will be sensitive to electronic symmetries and map them into the amplitude, phase and polarization of the emitted radiation. On the attosecond time scale, high-harmonic spectroscopy may become an important tool to investigate purely electronic dynamics. In the majority of polyatomic molecules, the orbital relaxation upon ionization is significant. In such cases, high-harmonic spectroscopy will offer a new approach to probing electron correlation - a property that is hard to measure by other means. We anticipate fruitful collaborations on both of these topics within NCCR MUST.

\section{Acknowledgements}

The work described in this article was performed at the National Research Council of Canada in collaboration with Mr. J. B. Bertrand, Dr. Y. Mairesse, Prof. P. B. Corkum and Dr. D. M. Villeneuve. Our current work in Zürich is supported by the Swiss Science Foundation under grant PP00P2_128274.

Received: March 5, 2011

[1] M. Drescher, M. Hentschel, R. Kienberger, M. Uiberacker, V. Yakovlev, A. Scrinzi, T. Westerwalbesloh, U. Kleineberg, U. Heinzmann, F. Krausz, Nature 2002, 419, 803.

[2] H. Niikura, F. Légaré, R. Hasbani, A. D. Bandrauk, M. Y. Ivanov, D. M. Villeneuve, P. B. Corkum, Nature 2002, 417, 917.

[3] P. Eckle, A. N. Pfeiffer, C. Cirelli, A. Staudte, R. Dörner, H. G. Muller, M. Büttiker, U. Keller, Science 2008, 322, 1525.

[4] O. Smirnova, Y. Mairesse, S. Patchkovskii, N. Dudovich, D. Villeneuve, P. Corkum, M. Y. Ivanov, Nature 2009, 460, 972.

[5] H. J. Wörner, J. B. Bertrand, P. Hockett, P. B. Corkum, D. M. Villeneuve, Phys. Rev. Lett. 2010, 104, 233904

[6] S. Haessler, J. Caillat, W. Boutu, C. GiovanettiTeixeira, T. Ruchon, T. Auguste, Z. Diveki, P. Breger, A. Maquet, B. Carré, R. Taïeb, P. Salières, Nat. Phys. 2010, 6, 200.
[7] E. Goulielmakis, Z.-H. Loh, A. Wirth, R. Santra, N. Rohringer, V. S. Yakovlev, S. Zherebtsov, T. Pfeifer, A. M. Azzeer, M. F. Kling, S. R. Leone, F. Krausz, Nature 2010, 466, 739.

[8] P. B. Corkum, Phys. Rev. Lett. 1993, 71, 1994.

[9] E. Goulielmakis, M. Schultze, M. Hofstetter, V. S. Yakovlev, J. Gagnon, M. Uiberacker, A. L. Aquila, E. M. Gullikson, D. T. Attwood, R. Kienberger, F. Krausz, U. Kleineberg, Science 2008, 320, 1614.

[10] H. Niikura, F. Légaré, R. Hasbani, M. Y. Ivanov, D. M. Villeneuve, P. B. Corkum, Nature 2003, $421,826$.

[11] M. Meckel, D. Comtois, D. Zeidler, A. Staudte, D. Pavičić, H. C. Bandulet, H. Pépin, J. C. Kieffer, R. Dörner, D. M. Villeneuve, P. B. Corkum, Science 2008, 320, 1478.

[12] M. Lein, N. Hay, R. Velotta, J. Marangos, P. Knight, Phys. Rev. Lett. 2002, 88, 183903.

[13] J. Itatani, J. Levesque, D. Zeidler, H. Niikura, H. Pépin, J. C. Kieffer, P. B. Corkum, D. M. Villeneuve, Nature 2004, 432, 867.

[14] T. Kanai, S. Minemoto, H. Sakai, Nature 2005, $435,470$.

[15] T. Morishita, A.-T. Le, Z. Chen, C. D. Lin, Phys. Rev. Lett. 2008, 100, 013903.

[16] H. J. Wörner, H. Niikura, J. B. Bertrand, P. B. Corkum, D. M. Villeneuve, Phys. Rev. Lett. 2009, 102, 103901.

[17] S. Baker, J. S. Robinson, C. A. Haworth, H. Teng, R. A. Smith, C. C. Chirilă, M. Lein, J. W. G. Tisch, J. P. Marangos, Science 2006, 312, 424.

[18] S. Baker, J. S. Robinson, M. Lein, C. C. Chirilă, R. Torres, H. C. Bandulet, D. Comtois, J. C. Kieffer, D. M. Villeneuve, J. W. G. Tisch, J. P. Marangos, Phys. Rev. Lett. 2008, 101, 053901.

[19] A.-T. Le, R. R. Lucchese, S. Tonzani, T. Morishita, C. D. Lin, Phys. Rev. A 2009, 80, 013401.

[20] A. D. Shiner et al., Nat. Phys. 2011, doi: 10.1038/nphys 1940 .

[21] Y. Mairesse, A. de Bohan, L. J. Frasinski, H. Merdji, L. C. Dinu, P. Monchicourt, P. Breger, M. Kovačev, R. Taïeb, B. Carré, H. G. Muller, P. Agostini, P. Salières, Science 2003, 302, 1540.

[22] G. Doumy, J. Wheeler, C. Roedig, R. Chirla, P. Agostini, L. F. DiMauro, Phys. Rev. Lett. 2009, 102, 093002.

[23] H. J. Wörner, J. B. Bertrand, D. V. Kartashov, P. B. Corkum, D. M. Villeneuve, Nature 2010, 466, 604.

[24] H. J. Wörner, J. B. Bertrand, P. B. Corkum, D. M. Villeneuve, Phys. Rev. Lett. 2010, 105, 103002.

[25] T. Kanai, E. J. Takahashi, Y. Nabekawa, K. Midorikawa, Phys. Rev. Lett. 2007, 98, 153904.

[26] H. J. Euchler, P. Günther, D. Pohl, 'LaserInduced Dynamic Gratings', Springer Verlag, 1986.

[27] Y. Mairesse, D. Zeidler, N. Dudovich, M. Spanner, J. Levesque, D. M. Villeneuve, P. B. Corkum, Phys. Rev. Lett. 2008, 100, 143903.

[28] B. K. McFarland, J. P. Farrell, P. H. Bucksbaum, M. Gühr, Science 2008, 322, 1232.

[29] M. V. Ammosov, N. B. Delone, V. P. Krainov, Zh. Eksp. Teor. Fiz. 1986, 91, 2008.

[30] J. Muth-Böhm, A. Becker, F. H. M. Faisal, Phys. Rev. Lett. 2000, 85, 2280.

[31] H. J. Wörner, P. B. Corkum, J. Phys. B: At. Mol. Opt. Phys. 2011, 44, 041001. 